\title{
Is persisting antigen responsible for the chronicity of experimental allergic arthritis?
}

\author{
A. FOX AND L. E. GLYNN
}

From the MRC Rheumatism Unit, Canadian Red Cross Memorial Hospital, Taplow, Maidenhead, Berkshir $\vec{B}$ SL6 OHN

SUMMARY Animals were injected intra-articularly with antigen after prior immunization with that antigen in Freund's incomplete adjuvant in order to precipitate immune complexes in the surfaces of menisci, ligaments, and cartilage. On reimmunization with antigen in Freund's complete adjuvan 10 weeks later, generally an arthritis limited to the intercondylar fossa developed; but on intrae articular injection of antigen a second time a widespread arthritis developed in that joint. Thus. immune deviation had not occurred and animals were in an immunological condition such as to be capable of developing widespread arthritis given the correct intra-articular stimulus. It is conclude⿻ that antigen, persisting as immune complexes, plays no part in maintaining widespread monarthritiso presumably owing to its inability to participate in a delayed hypersensitivity reaction as a result of sequestration.

Experimental allergic arthritis (EAA) is a chronic disease which histologically resembles rheumatoid arthritis. It is produced in rabbits by immunizing them with a soluble antigen in Freund's complete adjuvant (FCA) and then injecting the antigen intraarticularly. The substitution of Freund's incomplete adjuvant (FIA) for FCA results only in an acute inflammation on joint challenge with antigen (Dumonde and Glynn, 1962; Consden et al., 1971).

It has been shown that in EAA antigen persists as immune complexes in the superficial layers of menisci, ligaments, and cartilage (Cooke et al., 1972; Hollister and Mannik, 1974) (but not in synovial fluid: Belovic and Kinsella, 1973; or synovium: Doble et al., 1973), and it was suggested that slow release of antigen from them continuously activates complement which in turn causes the persisting inflammation. However, it has also been shown that immune complexes persist in similar locations in similar amounts in the joints of animals injected with antigen after prior immunization with that antigen in FIA. Since these joints recover completely within a few weeks from the induced arthritis, it was concluded that something more than immune complexes activating complement is required (Fox and Glynn, 1975).

Accepted for publication April 30, 1976

Correspondence to Mr. A. Fox
It was therefore suggested that slow release of antigen from complexes could continuously activate a delayed hypersensitivity (DHS) reaction. The neeके for a DHS reaction to antigen which is demonstrably not produced on immunization with antigen ip FIA but requires FCA (Audibert et al., 1976), has been stressed (Glynn, 1968). In addition the prof duction of a macrophage migration inhibition factor (MIF) by cultures of synovia from EAA joint suggests a continuing DHS reaction (Stastny, et al:1973). Also injection of MIF into joints results i arthritis (Andreis et al., 1974).

We present evidence that if animals are immunizeक्ष with antigen in FIA and then injected intra-articularly with that antigen in order to precipitate complexes in collagenous surfaces, on reimmunization wito antigen in FCA 10 weeks later they do not develop a widespread chronic arthritis although a milw inflammation does develop which is restricted to the intercondylar fossa. However, on intra-articular reinjection of antigen unequivocal arthritis does develop throughout the joint, showing that this immunization schedule does not induce immune deviation and that the animals are in an immunos logical condition to develop arthritis given the correct intra-articular stimulus. We conclude that antigew persisting as immune complexes is not involved in maintaining widespread arthritis, presumably as result of inability to participate in a DHS reaction. $\frac{2}{0}$ 


\section{Materials and methods}

Rabbits were of the old English strain bred in our own animal house. Ovalbumin (OA) was purchased from Koch-Light Ltd, Colnbrook, Bucks; FIA from Difco Laboratories, East Molesly, Surrey. Mycobacerium tuberculosis strains C, DT, and PN mixed were obtained from the Ministry of Agriculture, Fisheries and Food, Central Veterinary Laboratory, Weybridge, Surrey.

IMMUNIZATION SCHEDULE FOR EXPERIMENT A OA $20 \mathrm{mg} / \mathrm{ml}$ in saline was emulsified with an equal volume of FIA. $1.0 \mathrm{ml}$ was injected subcutaneously into each of 6 rabbits and repeated 3 weeks later. A second group of 8 rabbits was treated similarly except the FIA contained Myco. tuberculosis $2.0 \mathrm{mg} / \mathrm{ml}$ (FCA) (control group). 12 days later $1.0 \mathrm{ml}$ sterile saline containing $10 \mathrm{mg}$ OA was injected into the left joint of all 14 animals. One of the FIA group died of anaphylaxis next day. 8 weeks after joint challenge 2 of the control group were killed and the remaining 6 at 11 weeks, and left and right synovium removed for histology.

Ten weeks after joint challenge $1.0 \mathrm{ml}$ of an emulsion of equal parts of FCA and saline containing OA at $20 \mathrm{mg} / \mathrm{ml}$ was injected subcutaneously into each of the experimental group. This was repeated 3 weeks later. The animals were then observed for a further 8 weeks at which time the left and right joints were removed for histology.
IMMUNIZATION SCHEDULE FOR EXPERIMENT B OA $20 \mathrm{mg} / \mathrm{ml}$ in saline was emulsified with an equal volume of FIA. $1.0 \mathrm{ml}$ of this emulsion was injected subcutaneously into each of 12 rabbits. This was repeated 2 weeks later. 3 weeks later $1.0 \mathrm{ml}$ sterile saline containing $10 \mathrm{mg} \mathrm{OA}$ was injected into the left knee joint of each animal. 10 weeks later OA $20 \mathrm{mg} / \mathrm{ml}$ in saline was emulsified with an equal volume of FCA and $1.0 \mathrm{ml}$ injected subcutaneously, and was repeated 3 weeks later. 10 days later $1.0 \mathrm{ml}$ sterile saline containing $10 \mathrm{mg}$ OA was injected into the left knee joint of 6 of the animals (positive control group). The animals were observed for a further 8 weeks (except one of the experimental group which died 6 weeks earlier), when they were killed and left and right knee joints removed for histology.

\section{Results (Table)}

Table Pooled results of experiments $A$ and $B$

\begin{tabular}{llll}
\hline Grade of arthritis & $I$ & $I I$ & III \\
\hline $3+$ & 1 & 0 & 1 \\
$2+$ & 1 & 0 & 3 \\
$1+$ & 1 & 1 & 2 \\
\pm & 1 & 6 & 0 \\
0 & 2 & 4 & 2 \\
\hline
\end{tabular}

Groups I and II were immunized with OA/FIA, then injected intra/ articularly with OA. 10 weeks later they were immunized wit h OA FCA. Group I was joint challenged with antigen a second time but group II was not. Group III was immunized with OA/FCA, then injected intra-articularly with $\mathbf{O A}$.

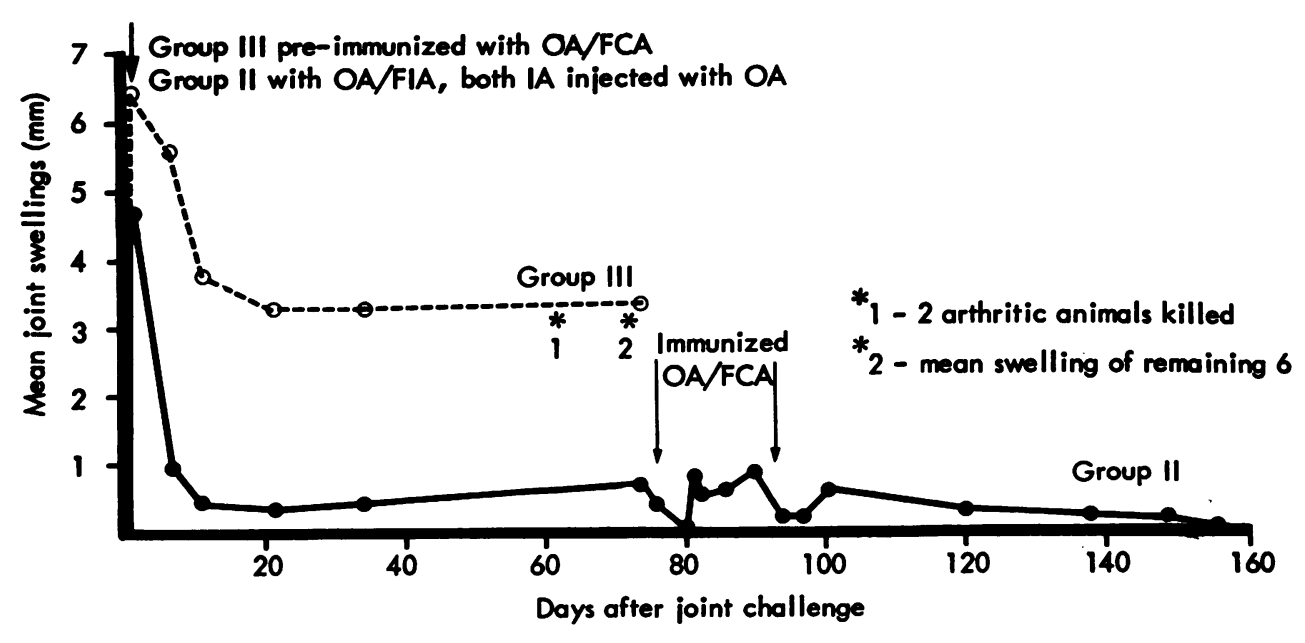

Fig. 1 Experiment A. Effect of immunization with (ovalbumin) $O A / F C A$ after prior precipitation of immune complexes in collagenous tissues. 


\section{HISTOLOGY}

The tissues fixed in formal saline were embedded in paraffin wax. Sections were cut at $7 \mu \mathrm{m}$ and stained with haematoxylin and eosin.

\section{ASSESSMENT OF ARTHRITIS}

Joints were measured regularly using an engineer's gauge and assessed histologically on a 0 to +++ grading.

\section{EXPERIMENT A (FIG. 1)}

On intra-articular challenge with antigen after prior immunization with the same antigen in FIA, animals developed an acute joint swelling similar to that seen in animals previously immunized with antigen in FCA. However, 6/8 immunized with FCA (control group) developed an unequivocal chronic arthritis which was confirmed histologically whereas none of the experimental group did. On reimmunization of the latter group with antigen in FCA 10 weeks after joint challenge, they still did not develop arthritis as assessed by joint measurements. However, on histological examination 2 showed clear plasma cell infiltration in the intercondylar fossa (Figs. 3 and 4) but not elsewhere. The other 3 showed no signs of chronic inflammation.

EXPERIMENT B (FIG. 2)

Similar findings occurred in this experiment. Namely, on intra-articular injection of antigen after prior immunization with FIA large joint swellings were믐 found at 24 hours, but had disappeared within $a$ few days. On reimmunization with antigen in FCA 10 weeks later no sign of arthritis occurred. However? 4 showed plasma cell infiltration in the intercon-0 dylar fossa, but not elsewhere and 1 which died $6 \mathrm{p}$ weeks before the end of the experiment did show widespread arthritis on histological examination of the autopsy specimen. Of the 6 joints rechallenged? with antigen, 3 developed signs of widespread arthritis which was confirmed histologically and developed a limited arthritis.

\section{Discussion and conclusions}

On intra-articular injection of antigen after prior $\vec{\omega}$ immunization with the same antigen in FIA, an acute arthritis develops which disappears in a fews days. In this situation immune complexes are deposited in the superficial layers of menisci, ligaments, and cartilage (Fox and Glynn, 1975).을 On reimmunization with antigen in FCA 10 weeks later joints generally do not develop widespread arthritis, but one limited to the intercondylar fossa. However, animals given this immunization schedule $\checkmark$ and the joint challenged a second time do generally develop widespread arthritis in the injected joint, showing that immune deviation has not occurred and animals are in an immunological condition such as to be capable of developing severe monarthritiso given the correct intra-articular stimulus.

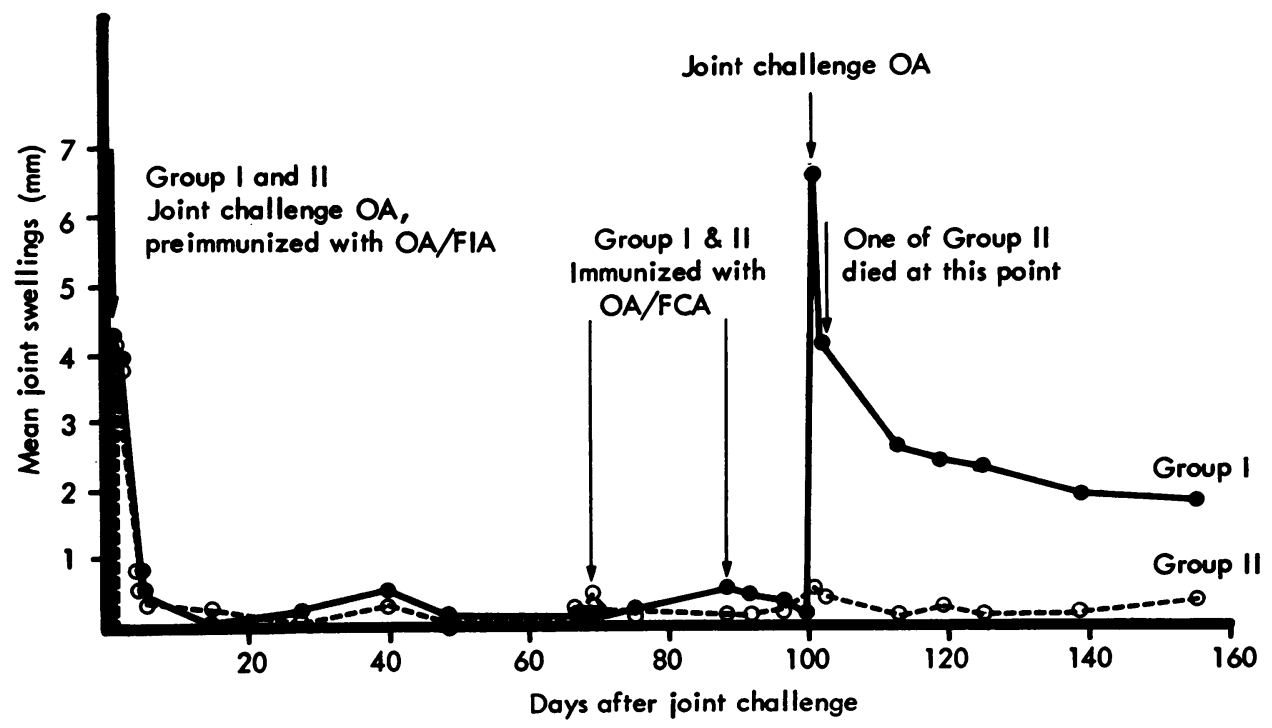

Fig. 2 Experiment B. Effect of immunization with $O A / F C A$ after prior precipitation of immune complexes in collagenous $\bar{D}$ tissues. 


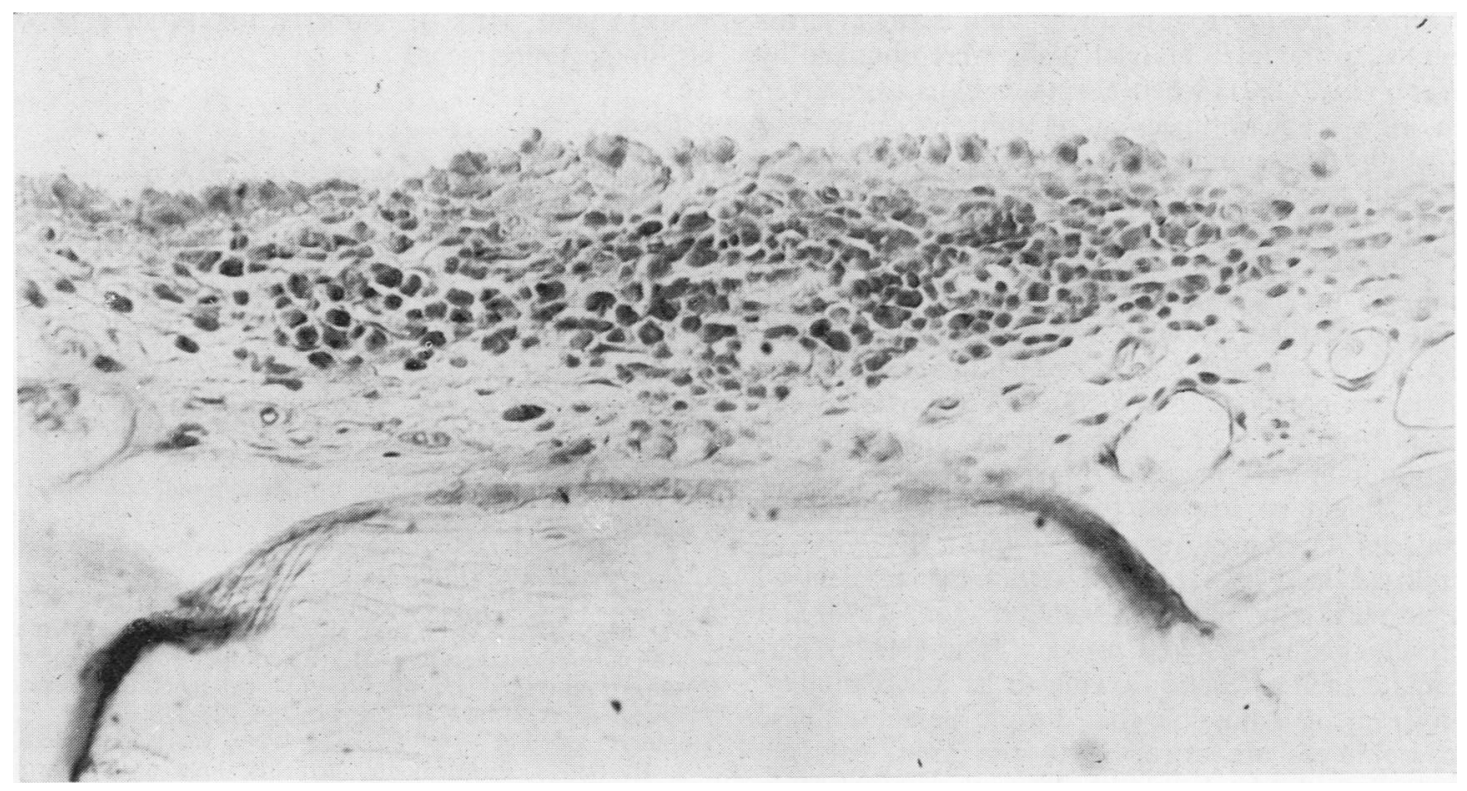

Fig. 3 Intercondylar fossa from the left knee joint of a rabbit immunized with $O A / F C A$, after prior precipitation of immune complexes in that joint. $\times 400$.

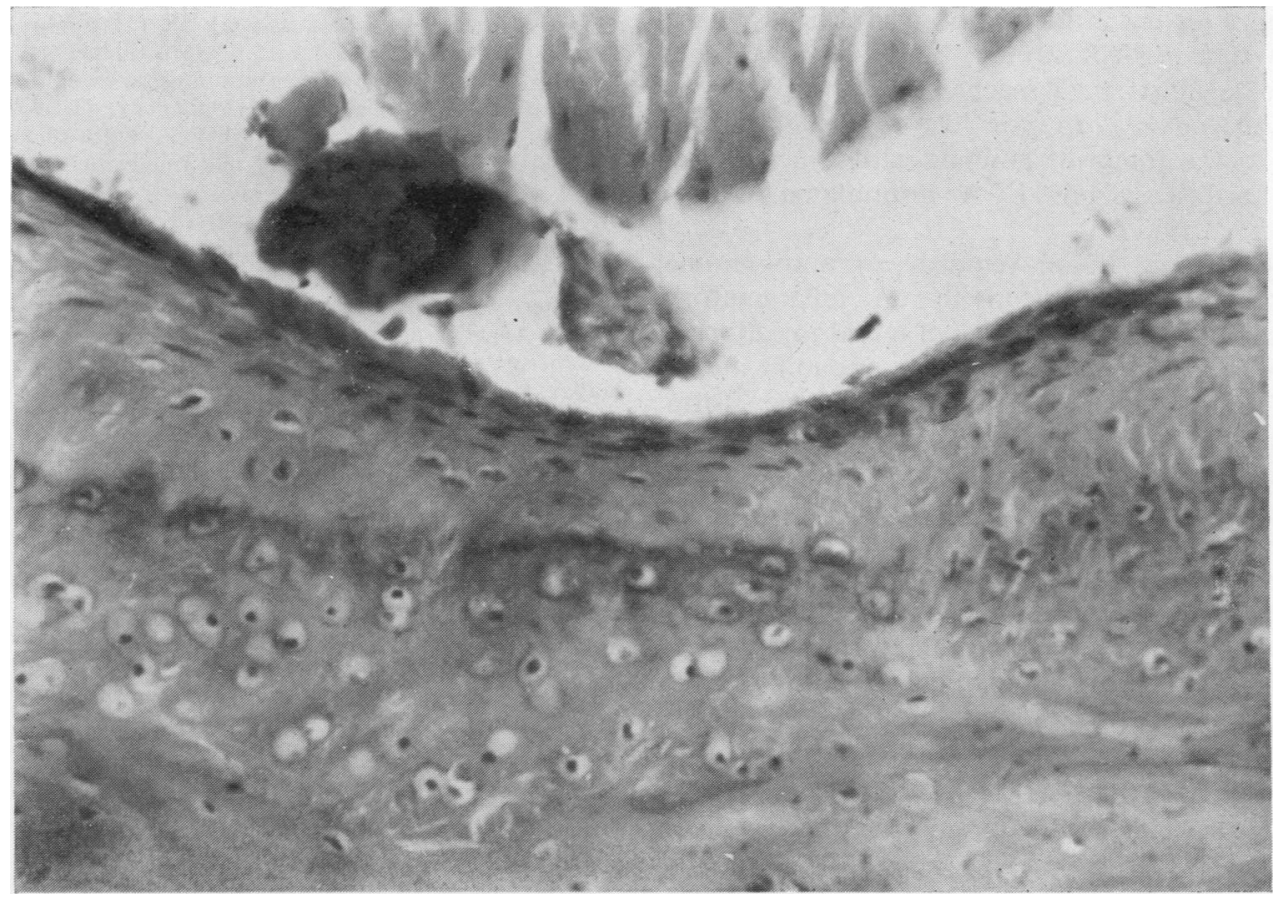

Fig. 4 Intercondylar fossa from the right knee joint of a rabbit immunized with $O A / F C A$, after prior precipitation of immune complexes in the left knee joint. $\times 330$. 
Similar results to our own, that is an arthritis limited to the intercondylar fossa, were obtained by Webb et al. (1971) when normal rabbits were given an intra-articular injection of antigen, followed 4 weeks later by immunization of the injected animals with the same antigen in FCA. Since antigen is almost totally lost from the joints of nonimmunized animals within a few weeks (Consden et al., 1971) it is unlikely that immune complexes were deposited in the avascular structures. Since these animals nevertheless developed an arthritis restricted to the intercondylar fossa, we conclude that such a limited arthritis can result from traces of antigen probably retained within or upon macrophages in this situation.

It should be stressed that the crucial difference between immunization with antigen in FCA, as opposed to FIA, is the development of delayed hypersensitivity reaction (Audibert et al., 1976) and presumably sequestered antigen is unable to perpetuate such a reaction owing to its inaccessibility. However, it is possible that during the acute phase macrophages are attracted to the joint, carrying with them dead tubercle bacilli or their breakdown products from the granuloma at the immunization site. Here they are released and might cause tissue damage in their own right or play the role of FCA in encouraging autoimmunization, either event resulting in further attraction of macrophages to the joint. We provided strong, but not conclusive, evidence against this mechanism, since neither 125I Myco. tuberculosis nor live Myco. avium are carried to the joints of animals with experimental allergic arthritis from FCA gramuloma depots (Doble et al., 1975).

If the chronic lesion requires an autoimmune reaction autoimmunization to an inflammatory product could arise at the time of initial injection of FCA. A similar product formed in the joint at the time of intra-articular challenge would then form the basis for self perpetuation. On immunization with various tissues in FCA autoimmune diseases are produced, e.g. whole brain (Kabat et al., 1947: experimental allergic encephalomyelitis) or whole testis (Voisin et al., 1951 : experimental allergic orchitis). It should be emphasized that these lesions tend to be self-limiting rather than self perpetuating. But with inflammatory exudate as the antigen, joint challenge with this same antigen, after prior immunization with FCA, can produce chronic, perpetuating lesions (Philips et al., 1966).

We thank Mrs J. Tyler, Mrs I. Blackman, and Mr A. Edwards for the photographic work; $\mathrm{Mr} \mathrm{J}$.
Watson and Miss J. Roberts for processing histological specimens.

\section{References}

Andreis, M., Stastny, P., and Ziff, M. (1974). Experimenta arthritis produced by injection of mediators of delayeg hypersensitivity. Arthritis and Rheumatism, 17, 537-552.

Audibert, F., Chedid, L., Lefrancier, P., and Choay, (1976). Distinctive adjuvanticity of synthetic analogs of mycobacterial water-soluble components. Cellular Immunology, 21, 243-249.

Belovic, B., and Kinsella, T. D. (1973). Immunofluorescent demonstration of an intra-articular antigen-antibod complex in experimental arthritis of the guinea pigit Annals of the Rheumatic Diseases, 32, 167-170.

Consden, R., Doble, A., Glynn, L. E., and Nind, A. P. (1971 w Production of a chronic arthritis with ovalbumin. It? retention in the rabbit knee joint. Annals of the Rheumatic Diseases, 30, 307-315.

Cooke, T. D., Hurd, E. R., Ziff, M., and Jasin, H. E. (1972) The pathogenesis of chronic inflammation in experimentaf antigen-induced arthritis. II. Preferential localization of antigen-antibody complexes to collagenous tissuesp Journal of Experimental Medicine, 135, 323-338.

Doble, A., Dorling, J., Glynn, L. E., Webb, J. A., and Wilcox J. H. (1973). Experimental arthritis, light and electro microscope studies of antigen and antibody using horseradish peroxidase as antigen. British Journal of Experimeno tal Pathology, 54, 646-651.

Doble, A., Fox, A., Glynn, L. E., and Kingston, D. (1975). The non-passage of mycobacteria from Freund's complete adjuvant granuloma depots to arthritic joints. Britis Journal of Experimental Pathology, 56, 537-543.

Dumonde, D. C., and Glynn, L. E. (1962). The production of arthritis in rabbits by an immunological reaction to fibring British Journal of Experimental Pathology, 43, 373-383.

Fox, A., and Glynn, L. E. (1975). Persistence of antigen nonarthritic joints. Annals of the Rheumatic Diseases, 34 431-437.

Glynn, L. E. (1968). The chronicity of inflammation and its significance in rheumatoid arthritis. Annals of the Rheuma tic Diseases, 27, 105-121.

Hollister, J. R., and Mannik, M. (1974). Antigen retention i joint tissues in antigen-induced synovitis. Clinical and Experimental Immunology, 16, 615-627.

Kabat, E. A., Wolf, A., and Bezer, A. E. (1947). The rapid production of acute disseminated encephalomyelitis iQ rhesus monkeys by injection of heterologous and homo logous brain tissue with adjuvants. Journal of Experimentab Medicine, 85, 117-130.

Philips, J. M., Kaklamanis, P., and Glynn, L. E. (1966) Experimental arthritis associated with auto-immunization. to inflammatory exudates. Annals of the Rheumatis Diseases, 25, 165-174.

Stastny, P., Cooke, T. D., and Ziff, M. (1973). Production of a macrophage migration inhibitory factor in rabbits with experimental arthritis. Clinical and Experimentado Immunology, 14, 141-147.

Voisin, G., Delaunay, A., and Barber, M. (1951). Sur des lesions testiculaires provoquées chez le cobaye par iso- ef auto-sensibilisation. Annales de l'Institut Pasteur, 81, 48 63.

Webb, F. W. S., Ford, P. M., and Glynn, L. E. (1971).0 Persistence of antigen in rabbit synovial membraneo British Journal of Experimental Pathology, 52, 31-35. 